\title{
Robust Speed Control for Networked DC Motor System
}

\author{
Sokliep Pheng ${ }^{1 *}$ \\ School of Information and Communication Engineering \\ Guilin University of Electronic Technology, Guilin, China
}

\author{
Luo Xiaonan ${ }^{2}$ \\ Guangxi Key Laboratory of Intelligent Processing of \\ Computer Image and Graphics \\ School of Computer Science and Information Security \\ Guilin University of Electronic Technology, Guilin, China
}

\author{
Rachana $\mathrm{Lav}^{3}$ \\ School of Energy Science and Engineering \\ University of Electronic Science and Technology \\ Chengdu, China
}

\author{
Zhongshuai Wang ${ }^{4}$, Zetao Jiang ${ }^{5}$ \\ School of Computer Science and Information Security \\ Guilin University of Electronic Technology \\ Guilin, China
}

\begin{abstract}
In this paper, we used observer-based $H_{\infty}$ output feedback control problems for the communication from the controller to dc motor and considered data packet dropout characterized by the Bernoulli random binary distribution the disturbance. The uncertain parameters have also been considered in the network-based dc motor system. Firstly, we used the robust $H_{\infty}$ output feedback control strategy to optimize controller gain and observer gain to guarantee the mean square stability. The observer-based $H_{\infty}$ output feedback has been designed to achieve robust speed control in the mean-square sense and optimize the parameters of the control system while guaranteeing the robust $H_{\infty}$ output feedback performance. Then, when data is transmitted in the control system, we illustrated that the system is stable and robust speed control can be achieved as well as the result realized.
\end{abstract}

Keywords-The Networked DC Motor System; Observer Design; Robust Speed Control; Data Dropout; LMI

\section{INTRODUCTION}

In recent years, robust speed control of NCS is the most population has been widely utilized in many industrial applications, and has very interesting in the industrial electronics community for control applications. Compared to traditional dc motor control, we found that the system component is located in the same place and connected by point-to-point wiring. Although, many requirements, dc motor, and controller are so hard to be located in the same place, and thus, signals are required to be transmitted from one place to another place. For improved networked control dc motor system technologies to reduce the cost of installation, easy maintenance [1], there is steady state error to integrate communication networked apply to the designed controller in the system. The systems are the type of NCS in the control system. Recently, NCSs is still popular and enticed much attention from research teams. To guarantee the obtaining good results such as networked control system, packet dropout, observer-design $\mathrm{H} \infty$ output feedback controller, and robust speed control have investigated with introduced in the literature [2].
The observer-designed $H_{\infty}$ output feedback control strategy is performed in the controller designed to corresponding robust speed control and determined controller gain and observer gain in the system. The data packets have transmitted through the network to the plant and there are some packet dropouts during networked transmissions. In this paper, the robust speed control proposed with observer designed and random data dropout have been considered and model to issue that in NCS is the packet losses phenomenon have solved in form mean-square sense. In addition, the previous work that studied about Markovian-jumping to find parameter was utilized to model a discrete-time as a linear system with data packet losses in NCSs [3] other literature research about random packet loss, observer-design $H_{\infty}$ feedback controller, robustly exponentially stabilize, prescribed disturbance rejection level in reference [4].

\section{RELATED WORK}

According to our research literature, we have focused and studied on two main points.

1) Observer designed to distribute: Generally, designed observer-based $H_{\infty}$ output feedback control strategy to optimize parameters performance, estimate state error, control the method utilized to achieve the exponentially stable in mean square sense for the network-based dc motor control system. This proposed is to archive robustly in the controller designed.

2) Robust speed control: After we got the observabilities, we also need robust speed control based on the exponentially stabilities mean square and robust $H_{\infty}$ feedback control strategy simultaneously for the NCS system. Therefore, our research purpose is to combine observabilities and robust $H_{\infty}$ feedback control strategy together which can be used for potential applications into the NCS system.

For controller designed in this paper we provide a scalar value $\gamma>0$. For the system in:

$\delta_{k+1}=\bar{A} \delta_{k}+\epsilon_{k} \tilde{A} \delta_{k}+\bar{B} \omega_{k}$

\footnotetext{
*Corresponding Author
} 
is exponentially stabilities mean square and we apply $H_{\infty}$ norm bound constraints [5-9]:

$$
\sum_{k=0}^{\infty} \mathrm{E}\left\{\left\|z_{k}\right\|^{2}\right\}<\gamma^{2} \sum_{k=0}^{\infty} \mathrm{E}\left\{\left\|\omega_{k}\right\|^{2}\right\}
$$

is obtained for all of nonzero $\omega_{k}$, if only if that there is the existence of positive definite in matrices $W_{11} \in R^{m \times m}, W_{22} \in R^{(n-m) \times(n-m)} \quad$, and $W_{2} \in R^{n \times p}$, and real matrices $Z \in R^{m \times n}$ and $N \in R^{n \times p}$, such as we proved in the systems (3) are represented [10]:

$\left[\begin{array}{cccccccccc}-W_{1} & * & * & * & * & * & * & * & * & * \\ 0 & -W_{2} & * & * & * & * & * & * & * & * \\ 0 & 0 & -\gamma^{2} \mathrm{I} & * & * & * & * & * & * & * \\ W_{1} A-\bar{\beta} B_{2} Z & \bar{\beta}_{3} Z & W_{1} B_{1} & -W_{1} & * & * & * & * & * & * \\ 0 & W_{2} A-Y C_{1} & W_{2} B_{1}-Y D_{2} & 0 & -W_{2} & * & * & * & * & * \\ 0 & 0 & 0 & 0 & 0 & -\mathrm{I} & * & * & * & * \\ \beta_{1} B_{2} Z & \beta_{1} B_{2} Z & 0 & 0 & 0 & 0 & -W_{1} & * & * & * \\ Y C_{1} & 0 & 0 & 0 & 0 & 0 & 0 & -W_{2} & * & * \\ 0 & 0 & 0 & H_{1}^{T} W_{1} & H_{1}^{T} W_{2} & 0 & 0 & 0 & -\in \mathrm{I} & * \\ \epsilon E & 0 & 0 & 0 & 0 & 0 & 0 & 0 & 0 & -\varepsilon \mathrm{I}\end{array}\right]<0$

$W_{1}:=U_{1}^{T} W_{11} U_{1}+U_{2}^{T} W_{22} U_{2}$

$U_{1}$, and $U_{2}$ it takes from the system (5). Moreover, to determine for the parameters are provided by the following system [11]:

$K=V \sum^{-1} W_{11}^{-1} \sum V^{T} Z, L=W_{2}^{-1} Y$

Proofs: $\quad$ Since there is the existing system $\quad W_{11}>0$ and $W_{22}>0$, such as:

$W_{1}=U_{1}^{T} W_{11} U_{1}+U_{2}^{T} W_{22} U_{2}$

Where

$U_{1}$ and $U_{2}$ are defined in the system (2.20) below:

$\tilde{B}=U B_{2} V=\left[\begin{array}{l}U_{1} \\ U_{2}\end{array}\right] B_{2} V=\left[\begin{array}{l}\Sigma \\ 0\end{array}\right]$

The following from the Lemma 1, and there are the exists of a nonsingular matrices

$W \in R^{m \times m}$, such as $B_{2} W=W_{1} B_{2}$

Now, let we computed such as a matrix $W$ that is related $B_{2} W=W_{1} B_{2}$ is following [12]:

$W_{1} U^{T}\left[\begin{array}{l}\Sigma \\ 0\end{array}\right] V^{T}=U^{T}\left[\begin{array}{c}\Sigma \\ 0\end{array}\right] V^{T} W$

And

$U^{T}\left[\begin{array}{cc}W_{11} & 0 \\ 0 & W_{22}\end{array}\right]\left[\begin{array}{l}\Sigma \\ 0\end{array}\right] V^{T}=U^{T}\left[\begin{array}{l}\Sigma \\ 0\end{array}\right] V^{T} W$
Which we are implied as:

$W=\left(V^{T}\right)^{-1} \Sigma^{-1} W_{11} \Sigma V^{T}$

For anyway, we can be integrated from (4) and (8) is that:

$B_{2} W=W_{1} B_{2}, Z=W K, Y=W_{2} L$

Furthermore, it is so hard to see that the system (3) is that equivalent to the equation (10) below [13-15]:

$\left[\begin{array}{cccccccccc}-W_{1} & * & * & * & * & * & * & * & * & * \\ 0 & -W_{2} & * & * & * & * & * & * & * & * \\ 0 & 0 & -\gamma^{2} \mathrm{I} & * & * & * & * & * & * & * \\ W_{1} A-\bar{\beta} W_{1} B_{2} K & \bar{\beta} W_{1} B_{2} K & W_{1} B_{1} & -W_{1} & * & * & * & * & * & * \\ 0 & W_{2} A-W_{2} L C_{1} & W_{2} B_{1}-W_{2} L & 0 & -W_{2} & * & * & * & * & * \\ 0 & 0 & 0 & 0 & 0 & -\mathrm{I} & * & * & * & * \\ \beta_{1} W_{1} B_{2} K & \beta_{1} W_{1} B_{2} K & 0 & 0 & 0 & 0 & -W_{1} & * & * & * \\ W_{2} L C_{1} & 0 & 0 & 0 & 0 & 0 & 0 & -W_{2} & * & * \\ 0 & 0 & 0 & H_{1}^{T} W_{1} & H_{1}^{T} W_{2} & 0 & 0 & 0 & -\epsilon \mathrm{I} & * \\ \epsilon E & 0 & 0 & 0 & 0 & 0 & 0 & 0 & 0 & -\varepsilon \mathrm{I}\end{array}\right]<0$

We considered the robust of $H_{\infty}$ control problems are computed for the type of NCS system with random distribution data dropout in the communication. In the introducing of the random packet losses and observer-design $H_{\infty}$ feedback control strategy to obtained robust speed control, exponentially stabilities mean square and we also obtained a prescribed $H_{\infty}$ performant for disturbance rejection level in control system. It is introduced that the controller problems are designed under considerations is computable if only if the LMI of the system (3) is able implied.

By the production, it takes out that the optimization problems have formulated as following in the system, the $H_{\infty}$ performant control strategy:

$\min _{W_{11}>0, W_{22}>0, Z, Y} \gamma$ Subject into the system (3)

Finally, after all of our proof that we are implemented the $H_{\infty}$ performance is obtained the control parameters in the control system.

\section{EXPERIMENTS AND SIMULATION}

\section{A. Test Simulation Results of the Stabilities}

This part of the simulation setup has considered on the network-based dc motor system, which the parameters are identifies from iNetCon-PC104 system. The simulation results of the network-based dc motor system under observer design to distributed into the system. The controller designed the system of closed-loop under observer based on $H_{\infty}$ output feedback control problems to optimize the system parameters. The $H_{\infty}$ performant control problems, which have been solved by LMI, by obtaining the strong of the robustness in the represented of the disturbance input for the closed-loop system. When the control signal has been transmitted, the packet losses rate is being considered in the NCS system and analysis the results are introduced of our method in this part. However, in the order of our studying for the random data 
dropout, observer design to distributed based on the $H_{\infty}$ output feedback control problems in the system to get the exponentially stabilities mean square and we can also obtain the prescribed of the $H_{\infty}$ disturbance rejection level. In addition, the system parameters of the network-based dc motor system are demonstrated.

The iNetCon-PC104 systems of the parameters are identified to get the brushless dc motor parameters in a transfer function form and then we obtained the system matrices as below:

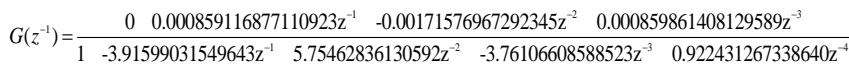

So it can be converted in the system matrices are obtained

$A=\left[\begin{array}{cccc}3.9160 & -5.7546 & 3.7611 & -0.9224 \\ 1 & 0 & 0 & 0 \\ 0 & 1 & 0 & 0 \\ 0 & 0 & 1 & 0\end{array}\right]$

$B_{2}=\left[\begin{array}{l}1 \\ 0 \\ 0 \\ 0\end{array}\right]$, and

$C_{1}=\left[\begin{array}{llll}0 & 0.0009 & -0.0017 & 0.0009\end{array}\right]$

$K=\left[\begin{array}{llll}4.6060 & -5.5768 & 3.7814 & -0.9215\end{array}\right]$

$L=\left[\begin{array}{llll}-511860 & -528080 & -471780 & -357940\end{array}\right]^{T}$

In the simulation, the networked dc motor system we considered which described by matrices as discrete-time modelling for the sampling period $\mathrm{t}=0.1 \mathrm{~s}$ and then we designed [40].

$H_{1}=\left[\begin{array}{llll}0.1 & 0 & 0 & 0\end{array}\right]^{T}, E=\left[\begin{array}{llll}1 & 0 & 0 & 0\end{array}\right], B_{1}=\left[\begin{array}{llll}0.5 & 0 & 0.2 & 0\end{array}\right]^{T}$

The disturbance input signal of this system is assumed that to be $\omega=1 / k$, and the time-varying of norm bound for the uncertain parameters $F(k)$ is supposed as following:

$F(k)=\left[\begin{array}{cccc}\text { rand } & 0 & 0 & 0 \\ 0 & \text { rand } & 0 & 0 \\ 0 & 0 & \text { rand } & 0 \\ 0 & 0 & 0 & \text { rand }\end{array}\right]$

In Fig. 1 as shown above, we used the observer designed to distribution in the network-based dc motor system for operation result and deal with random data dropout for the signals that it transmitted into the networked. In addition, we want to provide the estimate of the internal state output feedback of a given real system, and we have known that the state is necessary to realize in LMI method by using MATLAB toolbox. The observer designed to distribution based $H_{\infty}$ feedback control strategy, and we can obtained the controller gain, observer gain and prescribed the $H_{\infty}$ performant control strategy to achieve as in system (11). The structure designed in Fig. 1 as in the form for the system of closed-loop in the exponentially stabilities means square for the network-based dc motor system operation.

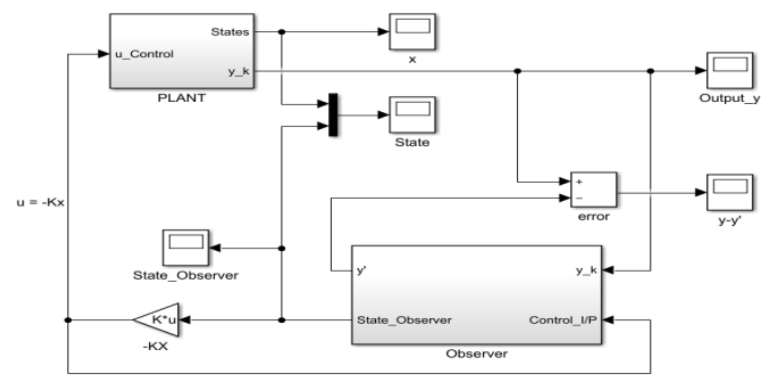

Fig. 1. Observer Design to Distribution.

\section{B. Packet Losses Rate}

In this addition, we have changed three value of $\bar{\beta}$ to performant in the simulation are given to demonstrate effectiveness for our studying method. We used to introduce the application for our method under observer designed to distribution based $H_{\infty}$ performant control strategy deals with random data dropout. In the simulation, results have represented to describe the possibilities and stabilities by using performant control strategy. In addition, we make the comparison for the control performant result with difference data dropout value, and we have been chosen by the difference data dropout value such as $\bar{\beta}=0.55,0.95$. Because of this applying of the following value in the simulation results shown by the figure as state response, control output, state estimate error and the packet losses respectively.

Step 1: We designed the $H_{\infty}$ performant control strategy with random for data dropout $\bar{\beta}=0.55$, and the $H_{\infty}$ performant is minimized as the index. Therefore, we greatly to an agreement with minimization of gamma problems. Solving of the $H_{\infty}$ optimization control strategy (11) by using MATLAB of the LMI toolbox, and we obtain the minimization of gamma $\gamma_{\text {min }}=0.8569$.

The initial condition of the uncertainty linear system of NCS is $x=\left[\begin{array}{llll}2 & 0 & 0 & 0\end{array}\right]$ and the observer initial condition is $\hat{x}=\left[\begin{array}{llll}0 & 0 & 0 & 0\end{array}\right]^{T}$, and the disturbance input we assumed that $\omega=1 / k$, the simulation results have shown below respectively.

Step 2: For the last step, we designed the $H_{\infty}$ performant control problems with appropriated random data dropout $\bar{\beta}=0.95$, and we are again considered with a suitable the $H_{\infty}$ performant $\gamma>0$ is minimized as the index. Solving of the $H_{\infty}$ optimization control problems (11) by using MATLAB of the LMI toolbox and we obtain a new of the minimization of gamma $\gamma_{\min }=0.4428$.

For the comparison of the simulation results as shown in above figures we can also see that when the larger value of 
$\bar{\beta}=0.95$ we got probabilities distribution of the data dropout is smaller and the network-based dc motor system performant is faster become to zero which means faster to be stable. In the order of this system to analysis for the simulation results, we choose the difference value of $\bar{\beta}$ to make a comparison in the system. The $\bar{\beta}$ represent is the probabilities of data dropout for the NCS system is converged to zero and the observer gain and controller gain are realized by applying the LMI approach in MATLAB toolbox.

According to the numerical of simulation results for the network-based dc motor system, we can see that in these terms, the performant $\gamma$ of observer design to distribution based on $H_{\infty}$ output feedback control problems is close to probabilities of the related for NCS system data dropout. We desired to achieve a minimum of that is solved in the LMI toolbox system by optimization of the $H_{\infty}$ performant control problem system in (11). So by using $\bar{\beta}=0.95$ we can conclude of the results that the probabilities of the NCS system data dropout are lower than other choose two value of $\bar{\beta}=0.55$, and the dc motor of NCS system performant is obtain in better condition. The analysis for the simulation results of this work is represented from Fig. 2 to Fig. 7, respectively.

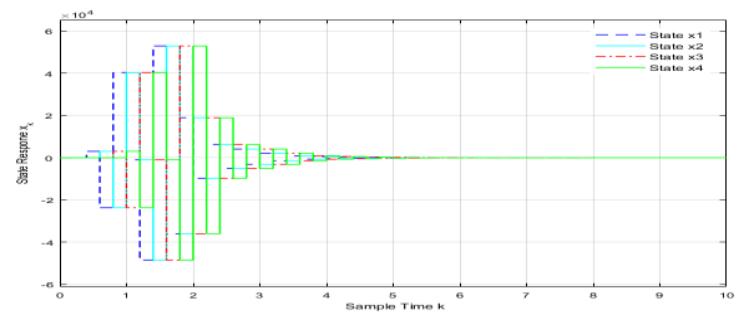

Fig. 2. Simulation of State Response $x_{k}$ with $\bar{\beta}=0.55$

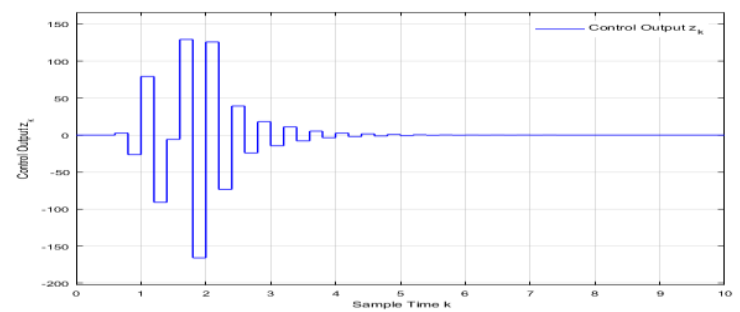

Fig. 3. Simulation of Control Output ${ }^{k}$ with $\bar{\beta}=0.55$

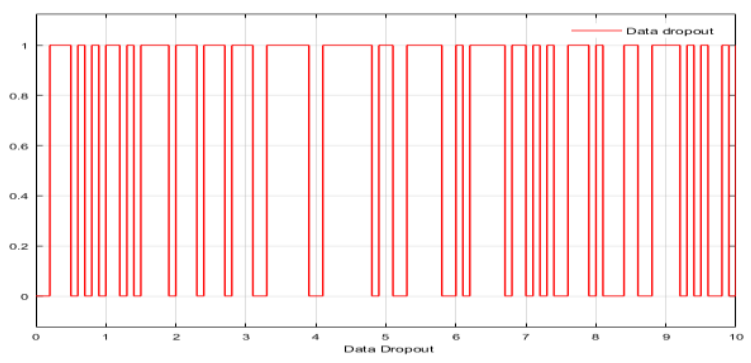

Fig. 4. Simulation of Data Dropout with $\bar{\beta}=0.55$

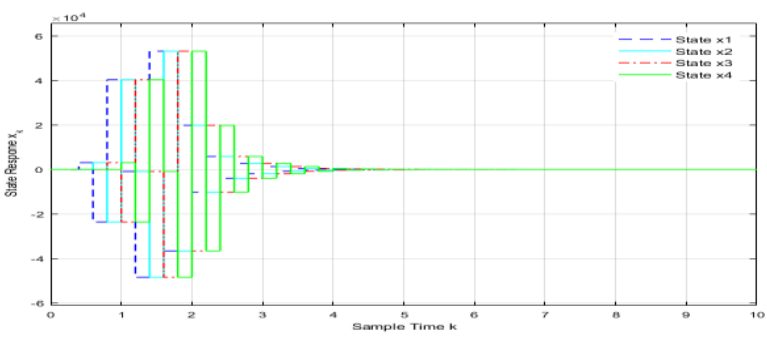

Fig. 5. Simulation of State Response $x_{k}$ with $\bar{\beta}=0.95$.

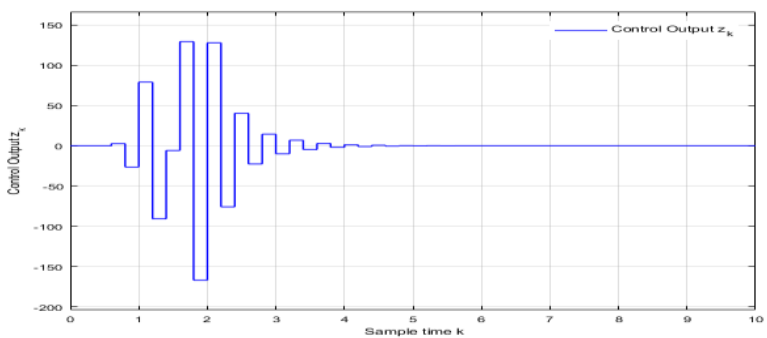

Fig. 6. Simulation of Control Output ${ }^{{ }_{k}}$ with $\bar{\beta}=0.95$.

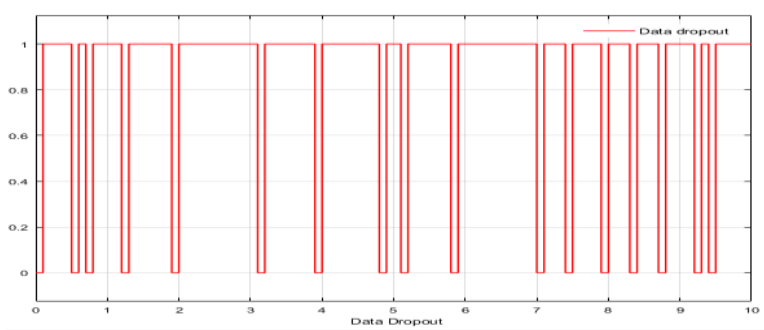

Fig. 7. Simulation of Data Dropout with $\bar{\beta}=0.95$

\section{Comparison Result with Previous Work}

The NCS system is very interesting, become more investigated and widely used in such an application, and there are many studies in the past. For development technology and improve previous results, we choose this topic to study and compare it to the previous work. The network-based dc motor system, designed observer-based $H_{\infty}$ performant control strategy deal with random data dropout is realized by the system of closed-loop is exponentially stabilities in the mean square sense that we can see in Fig. 8 become too stable faster than previous research are shown in Reference [11]. The simulation result has shown in Fig. 2 and Fig. 5 achieved by choosing three different values to make a comparison in this work. As above results, the value of $\bar{\beta}=0.95$ it became too stable faster than the other two values. The simulation results which have been given to introduce the state response, the state observer, data dropout and the control output $z(k)$ with disturbance rejection level are obtained in the system of closed-loop is exponentially stabilities in mean square sense are illustrated in Fig. 2 and Fig. 5. It is facile to show that when we considered of difference value the probabilities of the data dropout, the system of closed-loop become stable under we designed observer-based $H_{\infty}$ performant control strategy. When the packet losses rate is lower, it means that 
the network-based dc motor system has become faster to stable. It shows that the designed observer is the reconstruction state can be tracked the original state, and the state in the system is very good. However, it still has some lack off in our proposed method. The advantage of this work, the state response and the observer state are faster to stable and the estimate error state is faster go to zero. In Fig. 8, as shown for the effectiveness of our studying for a new design observer to distribute based $H_{\infty}$ performance strategy [11].

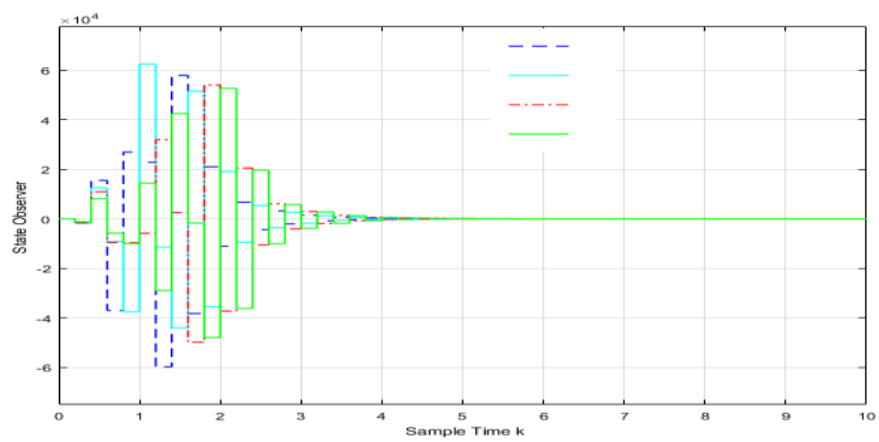

Fig. 8. Simulation of State Observer $\hat{x}$ with $\bar{\beta}=0.95$

According to Fig. 8, the simulation results which have been demonstrated of the effectiveness of our proposed method by using a new designed observer-based $H_{\infty}$ output feedback control strategy that the $H_{\infty}$ performant have realized by MATLAB of the LMI toolbox. After we solved the LMI we obtained the controller gain and the observer gain to implement in the system of closed-loop is exponentially stabilities of the mean square. Moreover, we have analysed our proposed method of a new designed observer-based $H_{\infty}$ performant control strategy by choosing the value of probabilities for random data dropout with $\bar{\beta}=0.95$. The initial condition, for uncertainly linear system of the NCS, is assumed as $x=\left[\begin{array}{llll}-4 & 4 & 0 & 0\end{array}\right]$ and the observer initial condition is also assumed as $\hat{x}=\left[\begin{array}{llll}0 & 0 & 0 & 0\end{array}\right]^{T}$, and the disturbance input we assumed that $\omega=1 / k$, for the simulation results that we have shown above respectively. The simulation results have become stable on $5 \mathrm{~s}$ which mean that the networked $\mathrm{dc}$ motor system is faster than the previous research are shown in [11]. For the previous works are stable on the 20s. Consequently, our results in above mentioned, it concluded that the observer-based robust $H_{\infty}$ feedback control strategy deal with random data dropout in the NCS system of closed-loop is robustly and exponentially stabilities in mean square sense to apply in the network-based dc motor system.

\section{RESULTS AND DISCUSSIONS}

\section{A. Simulation Result of Robust Speed Control}

After we got the stabilities of the system, robust speed control has been considered to apply for the network-based dc motor system. It is demonstrated the effectiveness of a new observer design based on $H_{\infty}$ performant control problems deal with random data dropout. We note that the network-based dc motor system has been designed to drive for the dc motor to prescribe the robust speed control. However, the observer designed is to optimize for the parameters and eliminated the steady-state tracking error, and the random data dropout is analysed about the data transmitted through the NCS system performant applied for dc motor. The specified of $H_{\infty}$ norm bound constraints in the system (3) have been solved in MATLAB of LMI toolbox.

The simulation by using iNetCon-PC104 system parameters with brushless dc motor, we are strongly focused about steady state speed tracking error under of our observer designed to distribute in the system that we have studied. The new system parameters of brushless dc motor that we identified from iNetCon-PC104 system to implement for the simulation results. The reference speed input we set $2000 \mathrm{rad} / \mathrm{s}$ in the simulation to illustrate the robust performance in the controller design. The effectiveness of our studied method by a new observer design to distribution based on $H_{\infty}$ performant control problems deal with random data dropout we have obtained the robust speed in the system performant. Now let we consider the terms to drive the network-based dc motor system to set the reference speed input $2000 \mathrm{rad} / \mathrm{s}$, we noted that under our proposed method is illuminated steady state speed error and the estimated state error and robust performance. To simulation of the network-based dc motor system, first we have considered on the system of closed-loop is exponentially stabilities mean square under using observer designed to distribution. The simulation results have done by implementing our method as well as shown:

In Fig. 9, the simulation result of brushless dc motor by communicated through networked with the iNetCon-PC104 system is robustly under using observer designed to distribute deal random data dropout into the system, the method want to achieve robust performance and stability. In the simulation result, we can succeed to control steady state speed tracking error and robustness of a network-based dc motor system under our proposed method, the robust speed performance is desirable with reference speed input $2000 \mathrm{rad} / \mathrm{s}$. However, the modelling of the network-based dc motor system, first, we have been proofed is exponentially stabilities mean square with uncertainty parameters and disturbance under new observer design to a distribution deal with data dropout. Second, the robust performance of networked dc motor system obtained after the above completed.

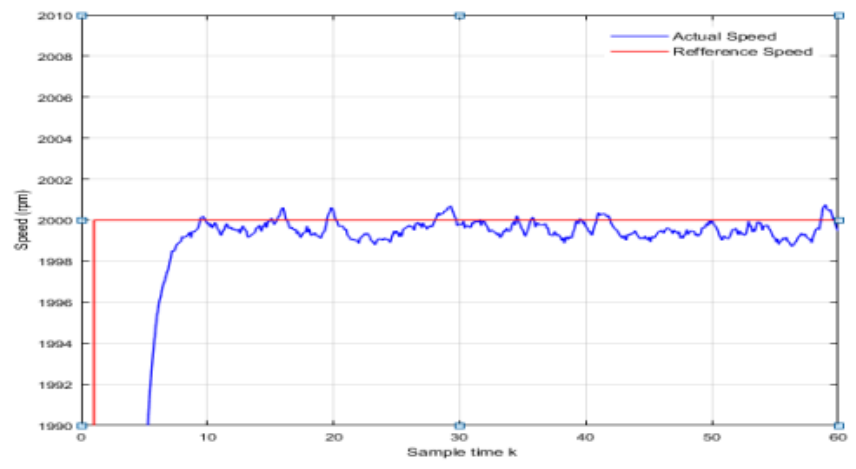

Fig. 9. Simulation Result of Robust Speed Control with Reference Speed 2000 Rpm. 


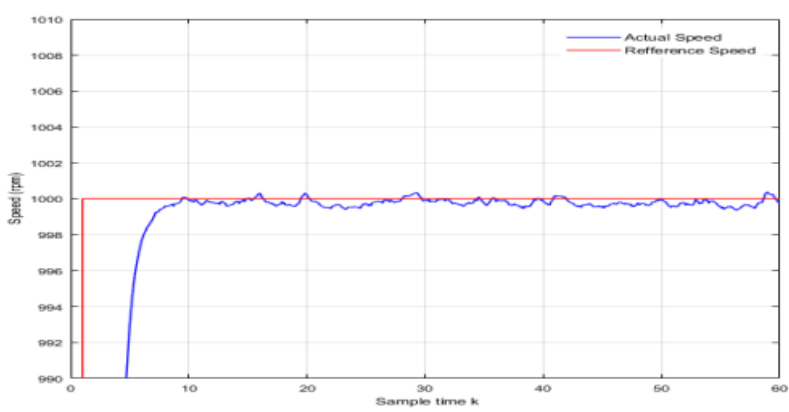

Fig. 10. Simulation Result of Robust Speed Control with Reference Speed 1000 Rpm.

After we settled reference speed $2000 \mathrm{rad} / \mathrm{s}$ and then we want to make simulation again with reference speed 1000 $\mathrm{rad} / \mathrm{s}$ to mention how is robust speed of the networked dc motor system by using observer design based $H_{\infty}$ output feedback controller. This simulation analysis is to demonstrate the robustness of the networked dc motor systems between the higher speed and the lower speed. The simulation result with reference speed $1000 \mathrm{rad} / \mathrm{s}$ have been done and evaluated in Fig. 10 is illustrated as following:

According to Fig. 10, the simulation result of the networked dc motor system demonstrated the speed error is eliminated and achieved the robust performance in the presence of bound modelling error. By using observer design based $H_{\infty}$ output feedback controller, the actual speed of networked dc motor system achieved robustness in the controller design. The simulation result can compare between the higher speed and lower speed so the reference speed is lower and the speed error of networked dc motor system is smaller too. Fig. 9 and Fig. 10 to demonstrated the robustness of the networked dc motor system in our proposed method.

\section{B. Experiment Result of Robust Speed Control}

In this part of work, we are mainly analysed of the characteristic of the experiment result of robust speed control of an NCS system. In the experimental, we used the iNetConPC104 system to communicate from the controller through the network and the objective is brushless dc motor. For the above part of work, we have done simulation successfully for the designed observer to distribution based on $H_{\infty}$ output feedback control problem. The experimental result of speed control for $\mathrm{dc}$ motor is robustly under our proposed method. Furthermore, we have experimented for the realization of brushless dc motor by the iNetCon-PC104 system to communicate through networked. The speed is robust which desirable under our proposed method.

For this part is very important to detail in the experiment which is the main part for our studied. It can be illustrated the speed that is robustly in the experimental result as well as figure:

In Fig. 11 above, we settled the reference speed input 2000, we saw that the speed was changed robustly under designed observer to distribution into the control system based on output feedback $H_{\infty}$ performant control problems. In the experimental result, we have successfully controlled the speed of a networked de motor system for our proposed method. It has shown how it becomes robustly with speed reference input. The effectiveness of our studying we can use observer designed to apply for the iNetCon-PC104 system can drive the brushless dc motor. Finally, the observer designed to optimize the parameters and eliminated the steady-state error, the robust speed control and analysed about the data transmitted through the NCS system performant by iNetCon-PC104 system which applied for brushless dc motor.

In this part, we want to make the experimental of networked dc motor system that we have changed the reference speed between 2000rand/s and $1000 \mathrm{rad} / \mathrm{s}$ in the communicated through network between controller and the plant to illustrate how to achieve robust performance from high speed to low speed and from low speed to high speed. The experimental of networked dc motor system has been demonstrated in the following figure:

According to Fig. 12, the red line is the reference speed and the black line is the actual speed of the networked dc motor system. The experimental results of the networked dc motor system that we settled reference speed $2000 \mathrm{rad} / \mathrm{s}$ and $1000 \mathrm{rad} / \mathrm{s}$ to achieve robust performance. By proposed our control strategy can be drive the speed of networked dc motor system from the high speed to low speed and from the low speed to the high speed to achieve robust speed control for the networked dc motor system. The experimental result demonstrated the effectiveness of our proposed method that we want to desired in the research strategy so the robust speed control for the networked dc motor system have been done by using our research strategy to make the simulation and the experimental result that we have already demonstrated in figure.

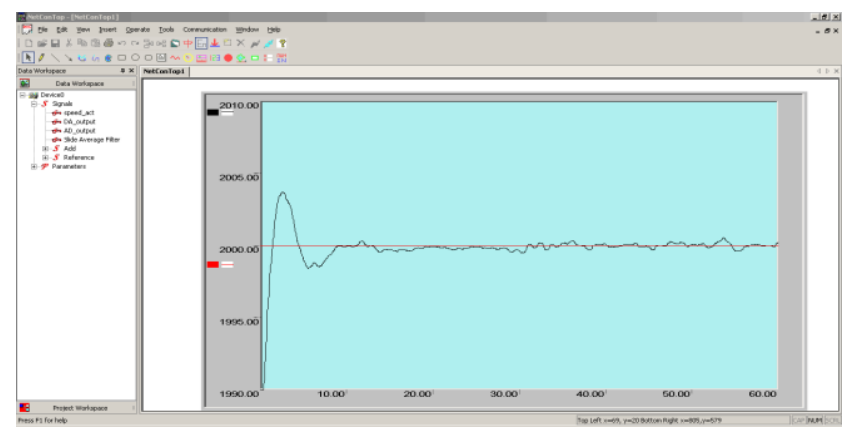

Fig. 11. Experiment Result of Robust Speed Control with Reference Speed 2000 Rpm.

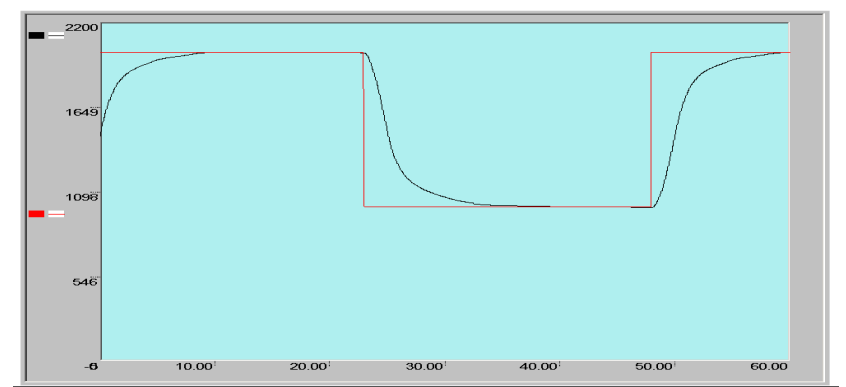

Fig. 12. Experiment Result of Robust Speed Control with Reference Speed 2000 and $1000 \mathrm{Rpm}$. 


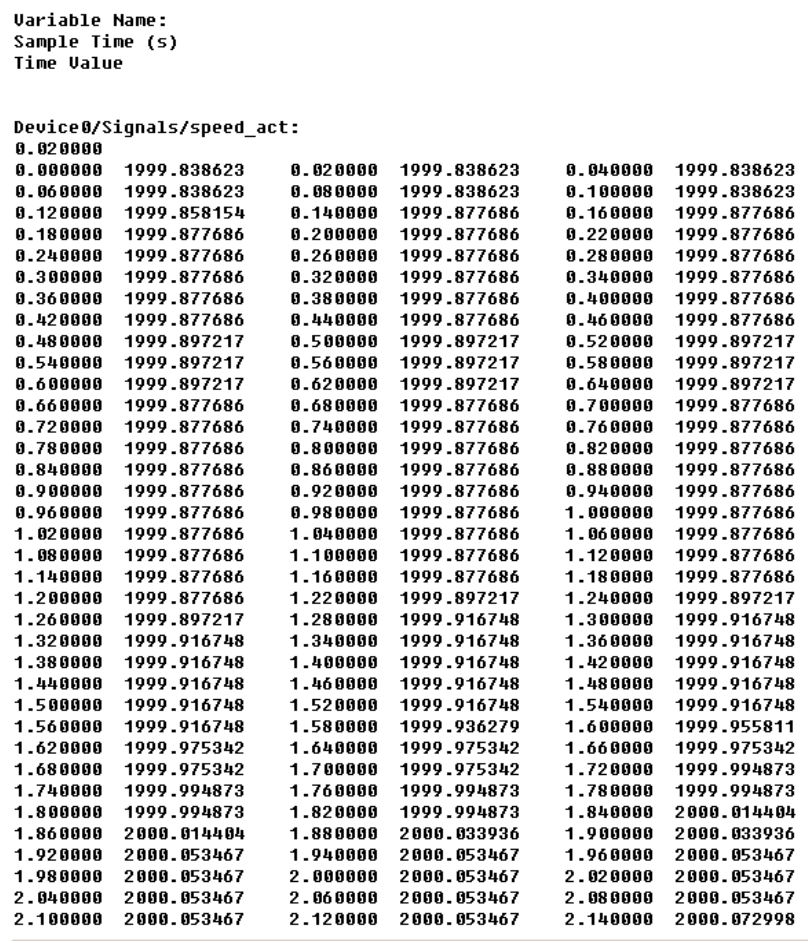

Fig. 13. Data of Speed Error.

After we did the experimental of the networked dc motor system to demonstrate the robustness, there are always some data speed error in the experimentation. The data speed error has been downloaded by using NetConTop software. The data steady state speed error of the networked dc motor system with iNetCon-PC104 to communicate through networked which the implement of the experimentation result is shown.

In Fig. 13, analysis of the experimental results of data steady state speed tracking error compared with reference input signals. The data have been saved from the iNetConnPC104 system by using NetconTop APP, and the hols system experiment we have used some software such as VMware Workstation to visual and communicated between controller to the plant, MATLAB R2208a to the designed structure of controller for NCS system and NetConTop to display the signal form input and the output of the system. Finally, the exponentially stabilities of the mean square and steady state speed tracking have been proofed and have been invested in our studying by a newly designed observer to distribution based on $H_{\infty}$ output feedback and consider with uncertainty parameter and disturbance.

\section{CONCLUSION}

Based on observer design to distribution consider on $H_{\infty}$ performant control problems deal with random data dropout, the exponentially stabilities of the mean square in the system of closed-loop, because of the unique advantages and wide potential application outlook have widely researched and developed. Compared to the previous research the NCS system stable result is lower than this work so far. However, the research on NCS system by applying the $H_{\infty}$ performant control problems which the LMI toolbox solving is still develop subsequently, it still has many unsolved problems, but now it has many simulations and experimental results studies on NCS system. This method can be also widely applied to the network-based dc motor system as well.

The creation of robust speed control for the network-based dc motor system, such as robust speed tracking error, the system of closed-loop is exponentially stabilities of the mean square. It has motivated and more inspiring for researcher to develop this kind of technology. Because the NCS system has transmitted data through a network without wiring, as well as to enrich robust speed control, and that is the reason of this paper uses a new observer design to distribution by $H_{\infty}$ performant to optimal parameters and improved the previous result as well.

In this paper, we have studied and illustrated observer designed to a distribution deal with random data dropout to performant into the networked dc motor system. Because of the data packets have transmitted in the control system and there is some packets dropout during network-based transmissions. In additions, the robust speed control proposed with observer designed and random data dropout has been considering and modeling to issue that in NCS system is the data dropout phenomenon have solved in the form of meansquare sense. Consequently, the network-based dc motor system can be able obtained by observer design to distribution with $H_{\infty}$ performant to optimize the parameters.

\section{ACKNOWLEDGMENT}

This work was supported in part by school of information and Communication Engineering of Guilin University of Electronic Technology and the National Natural Science Foundation of China (Nos. 61562016, 61702129, 61772149, and 61320106008), and by Guangxi Key Laboratory of Intelligent Processing of Computer Images and Graphics (No. GIIP201703).

\section{REFERENCES}

[1] Ren M, Zhang J, Jiang M, Yu M and Xu J 2015 Minimum(h, $\varphi)$-Entropy Control for Non-Gaussian Stochastic Networked Control Systems and Its Application to a Networked DC Motor Control System IEEE Trans. Control Syst. Technol. 23 406-11.

[2] Zhang X, Hengster-Movrić K, Šebek M, Desmet W and Faria C 2019 Distributed Observer and Controller Design for Spatially Interconnected Systems IEEE Trans. Control Syst. Technol. 27 1-13.

[3] Tang X, Deng L, Yu J and Qu H 2018 Output Feedback Predictive Control of Interval Type-2 T-S Fuzzy Systems With Markovian Packet Loss IEEE Trans. Fuzzy Syst. 26 2450-9.

[4] Xue B, Yu H and Wang M 2019 Robust Ho Output Feedback Control of Networked Control Systems With Discrete Distributed Delays Subject to Packet Dropout and Quantization IEEE Access 7 30313-20.

[5] Postoyan R, Wouw N van de, Nešić D and Heemels W P M H 2014 Tracking Control for Nonlinear Networked Control Systems IEEE Trans. Automat. Contr. 59 1539-54.

[6] Liu G, Yang Y and Yuan C 2013 Observer-based Hळ control for discrete-time systems with random communication delays 2013 25th Chinese Control and Decision Conference (CCDC) pp 3353-8.

[7] Liu S, Liu P X and Wang X $2016 \mathrm{H} \infty$ control of networked control systems with stochastic measurement losses 2016 IEEE International Conference on Information and Automation (ICIA) pp 1691-6.

[8] Zhang Q, Zhao D and Wang D 2018 Event-Based Robust Control for Uncertain Nonlinear Systems Using Adaptive Dynamic Programming IEEE Trans. Neural Networks Learn. Syst. 29 37-50. 
[9] Ho S, Chen C and Chen B 2017 Analysis for the RobustHo Synchronization of Nonlinear Stochastic Coupling Networks Through Poisson Processes and Core Coupling Design IEEE Trans. Control Netw. Syst. 4 223-35.

[10] Zhou Y and Hu S $2015 \mathrm{H}$ infinity control for DC servo motor in the network environment 2015 IEEE Advanced Information Technology, Electronic and Automation Control Conference (IAEAC) pp 401-5.

[11] Yu J, Yang C, Tang X and Wang P 2018 Ho control for uncertain linear system over networks with Bernoulli data dropout and actuator saturation ISA Trans. 74 1-13.

[12] Wang D, Liu D, Li H, Luo B and Ma H 2016 An Approximate Optimal Control Approach for Robust Stabilization of a Class of Discrete-Time
Nonlinear Systems With Uncertainties IEEE Trans. Syst. Man, Cybern. Syst. $46713-7$.

[13] Wang Z, Yang F, Ho D W C and Liu X 2007 RobustHo Control for Networked Systems With Random Packet Losses IEEE Trans. Syst. Man, Cybern. Part B 37 916-24.

[14] Li Z, Chang X and Wang Y 2017 Robust observer-based Hoo control for networked control systems with measurement quantization and packet dropouts 2017 12th IEEE Conference on Industrial Electronics and Applications (ICIEA) pp 739-44.

[15] Liu Y, Zhu X, Zhang H and Basin M 2018 Improved Robust Speed Tracking Controller Design for an Integrated Motor-Transmission Powertrain System Over Controller Area Network IEEE/ASME Trans. Mechatronics 23 1404-14. 